\title{
Annular Bounds for the Zeros of a Polynomial
}

\author{
Le Gao' ${ }^{1}$ and N. K. Govil (iD ${ }^{2}$ \\ ${ }^{1}$ Department of Mechanical Engineering, Auburn University, Auburn, AL 36849, USA \\ ${ }^{2}$ Department of Mathematics and Statistics, Auburn University, Auburn, AL 36849, USA
}

Correspondence should be addressed to N. K. Govil; govilnk@auburn.edu

Received 21 January 2018; Accepted 25 February 2018; Published 2 April 2018

Academic Editor: Irena Lasiecka

Copyright (C) 2018 Le Gao and N. K. Govil. This is an open access article distributed under the Creative Commons Attribution License, which permits unrestricted use, distribution, and reproduction in any medium, provided the original work is properly cited.

The problem of obtaining the smallest possible region containing all the zeros of a polynomial has been attracting more and more attention recently, and in this paper, we obtain several results providing the annular regions that contain all the zeros of a complex polynomial. Using MATLAB, we construct specific examples of polynomials and show that for these polynomials our results give sharper regions than those obtainable from some of the known results.

\section{Introduction}

The Fundamental Theorem of Algebra states that every nonzero, single-variable, polynomial

$$
p(z)=a_{0}+a_{1} z+a_{2} z^{2}+a_{3} z^{3}+\cdots+a_{n} z^{n},
$$

of degree $n$ with complex coefficients, has exactly $n$ complex zeros. The zeros may however be coincident. Although the above theorem tells about the number of zeros, it does not mention anything about the location of these zeros. The problem of obtaining the smallest possible region containing all the zeros of a polynomial has been attracting more and more attention recently, since the results are very useful in engineering applications as well as in many areas of applied mathematics such as control theory, cryptography, mathematical biology, and combinatorics [1-4].

For any second-degree polynomial equation, $a x^{2}+b x+$ $c=0, a \neq 0$, the roots can be found by using the familiar quadratic formula, $x=\left(-b \pm \sqrt{b^{2}-4 a c}\right) / 2 a$, and for polynomials of third and fourth degree, there are analogous formulas to find the zeros. However, for polynomials of degree five or higher with arbitrary coefficients, the AbelRuffini theorem states that there is no algebraic solution. Several methods, for example, Aberth-Ehrlich method [5, 6], have been proposed for the simultaneous determination of zeros of algebraic polynomials and there are studies $[7,8]$ to accelerate convergence and increase computational efficiency of these methods. Approximations to the zeros of a polynomial can be drawn by these methods, and these methods can become more efficient when an annulus containing all the zeros of the polynomial is provided. So this paper is focused on finding new theorems that can provide smaller annuli containing all the zeros of a polynomial.

\section{Preliminaries}

The first contributor to this subject was probably Gauss, who proved the following.

Theorem 1 (Gauss). A polynomial

$$
p(z)=a_{0}+a_{1} z+a_{2} z^{2}+a_{3} z^{3}+\cdots+a_{n} z^{n},
$$

with all $a_{k}$ real, has no zeros outside the circle $|z|=R$, where

$$
R=\max _{1 \leq k \leq n}\left(n \sqrt{2}\left|a_{k}\right|\right)^{1 / k} .
$$

Later in 1850, Gauss [9] proved that, for any real or complex $a_{k}$, the radius $R$ may be taken as the positive root of the equation

$$
z^{n}-2^{1 / 2}\left(\left|a_{1}\right| z^{n-1}+\cdots+\left|a_{n}\right|\right)=0 .
$$

Theorem 1 of Gauss was improved in 1829 by Cauchy [10] who derived more exact bounds for the moduli of the zeros 
of a polynomial than those given by Gauss, by proving the following.

Theorem 2 (Cauchy). If

$$
p(z)=a_{0}+a_{1} z+a_{2} z^{2}+a_{3} z^{3}+\cdots+a_{n} z^{n}
$$

is a complex polynomial of degree $n$, then all the zeros lie in the disc

$$
\{z:|z|<\eta\} \subset\{z:|z|<1+A\}
$$

where $A=\max _{1 \leq k \leq n-1}\left|a_{k}\right|$ and $\eta$ is the unique positive root of the real-coefficient equation

$$
z^{n}-\left|a_{n-1}\right| z^{n-1}-\left|a_{n-2}\right| z^{n-2}-\cdots-\left|a_{1}\right| z-\left|a_{0}\right|=0 \text {. }
$$

By applying Theorem 2 to the polynomial $p(z)=z^{n} p(1 / z)$, we easily get Theorem 3.

Theorem 3. All the zeros of the polynomial

$$
p(z)=a_{0}+a_{1} z+a_{2} z^{2}+a_{3} z^{3}+\cdots+a_{n} z^{n}, \quad a_{n} \neq 0,
$$

lie in the annulus $r_{1} \leq|z| \leq r_{2}$, where $r_{1}$ is the unique positive root of the equation

$$
\begin{aligned}
& \left|a_{n}\right| z^{n}+\left|a_{n-1}\right| z^{n-1}+\left|a_{n-2}\right| z^{n-2}+\cdots+\left|a_{1}\right| z-\left|a_{0}\right| \\
& \quad=0
\end{aligned}
$$

and $r_{2}$ is the unique positive root of the equation

$$
\left|a_{0}\right|+\left|a_{1}\right| z+\cdots+\left|a_{n-1}\right| z^{n-1}-\left|a_{n}\right| z^{n}=0 .
$$

Diaz-Barrero [11] gave the following results, providing circular regions containing all the zeros of a polynomial in terms of the binomial coefficients and Fibonacci's numbers. Note that the binomial coefficients are defined by

$$
C(n, k)=\frac{n !}{k !(n-k) !}, \quad 0 !=1,
$$

and Fibonacci's numbers are defined by

$$
\begin{aligned}
& F_{0}=0, \\
& F_{1}=1, \\
& F_{j}=F_{j-1}+F_{j-2}, \text { for } j \geq 2 .
\end{aligned}
$$

Theorem 4. Let $p(z)=\sum_{j=0}^{n} a_{j} z^{j}\left(a_{j} \neq 0,0 \leq j \leq n\right)$ be a nonconstant complex polynomial. Then all its zeros lie in the $\operatorname{disc} C=\left\{z: r_{1} \leq|z| \leq r_{2}\right\}$, where

$$
\begin{aligned}
& r_{1}=\frac{3}{2} \min _{1 \leq j \leq n}\left\{\frac{2^{n} F_{j} C_{j}^{n}}{F_{4 n}}\left|\frac{a_{0}}{a_{j}}\right|\right\}^{1 / k}, \\
& r_{2}=\frac{2}{3} \max _{1 \leq j \leq n}\left\{\frac{F_{4 n}}{2^{n} F_{j} C_{j}^{n}}\left|\frac{a_{n-j}}{a_{n}}\right|\right\}^{1 / k} .
\end{aligned}
$$

Kim [12] provides another annulus containing all the zeros of a polynomial with the binomial coefficients.

Theorem 5. Let $p(z)=\sum_{k=0}^{n} a_{k} z^{k}\left(a_{k} \neq 0,0 \leq k \leq n\right)$ be $a$ nonconstant polynomial with complex coefficients. Then all the zeros of $p(z)$ lie in the annulus $A=\left\{z: r_{1} \leq|z| \leq r_{2}\right\}$, where

$$
\begin{aligned}
& r_{1}=\min _{1 \leq k \leq n}\left\{\frac{C(n, k)}{2^{n}-1}\left|\frac{a_{0}}{a_{k}}\right|\right\}^{1 / k}, \\
& r_{2}=\max _{1 \leq k \leq n}\left\{\frac{2^{n}-1}{C(n, k)}\left|\frac{a_{n-k}}{a_{n}}\right|\right\}^{1 / k} .
\end{aligned}
$$

There are in fact many results in this direction, and for some more results, see, for example, [13, 14].

Recently, Dalal and Govil [15] unified the above theorems and proved the following theorem.

Theorem 6. Let $A_{k}>0$ for $1 \leq k \leq n$ such that $\sum_{k=1}^{n} A_{k}=1$. If $p(z)=a_{0}+a_{1} z+a_{2} z^{2}+a_{3} z^{3}+\cdots+a_{n} z^{n}$ is a nonconstant complex polynomial of degree $n$, with $a_{k} \neq 0$ for $1 \leq k \leq n$, then all the zeros of $p(z)$ lie in the annulus $C=\left\{z: r_{1} \leq|z| \leq r_{2}\right\}$, where

$$
\begin{aligned}
& r_{1}=\min _{1 \leq k \leq n}\left\{A_{k}\left|\frac{a_{0}}{a_{k}}\right|\right\}^{1 / k}, \\
& r_{2}=\max _{1 \leq k \leq n}\left\{\frac{1}{A_{k}}\left|\frac{a_{n-k}}{a_{n}}\right|\right\}^{1 / k} .
\end{aligned}
$$

Using the above theorems, Dalal and Govil [15] also gave the following.

Theorem 7. Let $p(z)=a_{0}+a_{1} z+a_{2} z^{2}+a_{3} z^{3}+\cdots+a_{n} z^{n}$ be a nonconstant complex polynomial of degree $n$, with $a_{k} \neq 0$ for $1 \leq k \leq n$. Then all the zeros of $p(z)$ lie in the annulus $C=\left\{z: r_{1} \leq|z| \leq r_{2}\right\}$, where

$$
\begin{aligned}
& r_{1}=\min _{1 \leq k \leq n}\left\{\frac{C_{k-1} C_{n-k}}{C_{n}}\left|\frac{a_{0}}{a_{k}}\right|\right\}^{1 / k}, \\
& r_{2}=\max _{1 \leq k \leq n}\left\{\frac{C_{n}}{C_{k-1} C_{n-k}}\left|\frac{a_{n-k}}{a_{n}}\right|\right\}^{1 / k},
\end{aligned}
$$

where

$$
C_{k}=\frac{C(2 k, k)}{k+1}
$$

is the kth Catalan number in which $C(2 k, k)$ are the binomial coefficients.

Theorem 6 of Dalal and Govil [15] can generate infinitely many results, including Theorems 4 and 5, giving annulus containing all the zeros of a polynomial, and over the years, mathematicians have shown the usefulness of their results by comparing their bounds with the existing bounds in the literature by giving some examples and thus showing that their bounds are better in some special cases. In this 
connection, Dalal and Govil in [16] have shown that no matter what result you obtain as a corollary to Theorem 6 , one can always generate polynomials for which the corollary so obtained gives better bound than the existing ones, implying that every result obtained by Theorem 6 can be useful. Since the results obtained as corollaries of Theorem 6 cannot in general be compared, more recently Dalal and Govil [17] have given results that help to compare the bounds for a subclass of polynomials. For this, they provide a class of polynomials with some conditions on degree or absolute range of coefficients of the polynomial, and for this class of polynomials, the bound obtained by one corollary is always better than the bound obtained from the other.

\section{Main Results and Their Proofs}

In this section, we obtain some new results which provide annuli containing all the zeros of a polynomial. These results have been obtained by making use of some identities and Theorem 6. Then, in Section 4, we use MATLAB to obtain examples of polynomials for which our results give bounds that are sharper than those obtainable from Theorems 5 and 7. In fact, it is not difficult to construct polynomials for which our theorems also give better bounds than those obtainable from Theorem 4.

Theorem 8. Let $p(z)=a_{0}+a_{1} z+a_{2} z^{2}+a_{3} z^{3}+\cdots+a_{n} z^{n}$ be a nonconstant complex polynomial of degree $n$, with $a_{k} \neq 0$ for $1 \leq k \leq n$. Then all the zeros of $p(z)$ lie in the annulus $C=\left\{z: r_{1} \leq|z| \leq r_{2}\right\}$, where

$$
\begin{aligned}
& r_{1}=\min _{1 \leq k \leq n}\left\{\frac{k C(n, k)^{2}}{n(n-k+1) C_{n}}\left|\frac{a_{0}}{a_{k}}\right|\right\}^{1 / k}, \\
& r_{2}=\max _{1 \leq k \leq n}\left\{\frac{n(n-k+1) C_{n}}{k C(n, k)^{2}}\left|\frac{a_{n-k}}{a_{n}}\right|\right\}^{1 / k} .
\end{aligned}
$$

Theorem 9. Let $p(z)=a_{0}+a_{1} z+a_{2} z^{2}+a_{3} z^{3}+\cdots+a_{n} z^{n}$ be a nonconstant complex polynomial of degree $n$, with $a_{k} \neq 0$ for $1 \leq k \leq n$. Then all the zeros of $p(z)$ lie in the annulus $C=\left\{z: r_{1} \leq|z| \leq r_{2}\right\}$, where

$$
\begin{aligned}
& r_{1}=\min _{1 \leq k \leq n}\left\{\frac{C(n, 2 k) C_{k} 2^{n-2 k}}{C_{n+1}-2^{n}}\left|\frac{a_{0}}{a_{k}}\right|\right\}^{1 / k}, \\
& r_{2}=\max _{1 \leq k \leq n}\left\{\frac{C_{n+1}-2^{n}}{C(n, 2 k) C_{k} 2^{n-2 k}}\left|\frac{a_{n-k}}{a_{n}}\right|\right\}^{1 / k} .
\end{aligned}
$$

Theorem 10. Let $p(z)=a_{0}+a_{1} z+a_{2} z^{2}+a_{3} z^{3}+\cdots+a_{n} z^{n}$ be a nonconstant complex polynomial of degree $n$, with $a_{k} \neq 0$ for $1 \leq k \leq n$. Then all the zeros of $p(z)$ lie in the annulus $C=\left\{z: r_{1} \leq|z| \leq r_{2}\right\}$, where

$$
\begin{aligned}
& r_{1}=\min _{1 \leq k \leq n}\left\{\frac{F_{2 k}}{F_{2 n+1}-1}\left|\frac{a_{0}}{a_{k}}\right|\right\}^{1 / k}, \\
& r_{2}=\max _{1 \leq k \leq n}\left\{\frac{F_{2 n+1}-1}{F_{2 k}}\left|\frac{a_{n-k}}{a_{n}}\right|\right\}^{1 / k} .
\end{aligned}
$$

Theorem 11. Let $p(z)=a_{0}+a_{1} z+a_{2} z^{2}+a_{3} z^{3}+\cdots+a_{n} z^{n}$ be a nonconstant complex polynomial of degree $n$, with $a_{k} \neq 0$ for $1 \leq k \leq n$. Then all the zeros of $p(z)$ lie in the annulus $C=\left\{z: r_{1} \leq|z| \leq r_{2}\right\}$, where

$$
\begin{aligned}
& r_{1}=\min _{1 \leq k \leq n}\left\{\frac{F_{k}^{2}}{F_{n} F_{n+1}}\left|\frac{a_{0}}{a_{k}}\right|\right\}^{1 / k}, \\
& r_{2}=\max _{1 \leq k \leq n}\left\{\frac{F_{n} F_{n+1}}{F_{k}^{2}}\left|\frac{a_{n-k}}{a_{n}}\right|\right\}^{1 / k} .
\end{aligned}
$$

Theorem 12. Let $p(z)=a_{0}+a_{1} z+a_{2} z^{2}+a_{3} z^{3}+\cdots+a_{n} z^{n}$ be a nonconstant complex polynomial of degree $n$, with $a_{k} \neq 0$ for $1 \leq k \leq n$. Then all the zeros of $p(z)$ lie in the annulus $C=\left\{z: r_{1} \leq|z| \leq r_{2}\right\}$, where

$$
\begin{aligned}
& r_{1}=\min _{1 \leq k \leq n}\left\{\frac{k^{2} C(n, k)}{n(n+1) 2^{n-2}}\left|\frac{a_{0}}{a_{k}}\right|\right\}^{1 / k}, \\
& r_{2}=\max _{1 \leq k \leq n}\left\{\frac{n(n+1) 2^{n-2}}{k^{2} C(n, k)}\left|\frac{a_{n-k}}{a_{n}}\right|\right\}^{1 / k} .
\end{aligned}
$$

Theorem 13. Let $p(z)=a_{0}+a_{1} z+a_{2} z^{2}+a_{3} z^{3}+\cdots+a_{n} z^{n}$ be a nonconstant complex polynomial of degree $n$, with $a_{k} \neq 0$ for $1 \leq k \leq n$. Then all the zeros of $p(z)$ lie in the annulus $C=\left\{z: r_{1} \leq|z| \leq r_{2}\right\}$, where

$$
\begin{aligned}
& r_{1}=\min _{1 \leq k \leq n}\left\{\frac{k C(n, k)^{2}}{(n / 2) C(2 n, n)}\left|\frac{a_{0}}{a_{k}}\right|\right\}^{1 / k}, \\
& r_{2}=\max _{1 \leq k \leq n}\left\{\frac{(n / 2) C(2 n, n)}{k C(n, k)^{2}}\left|\frac{a_{n-k}}{a_{n}}\right|\right\}^{1 / k} .
\end{aligned}
$$

Theorem 14. Let $p(z)=a_{0}+a_{1} z+a_{2} z^{2}+a_{3} z^{3}+\cdots+a_{n} z^{n}$ be a nonconstant complex polynomial of degree $n$, with $a_{k} \neq 0$ for $1 \leq k \leq n$. Then all the zeros of $p(z)$ lie in the annulus $C=\left\{z: r_{1} \leq|z| \leq r_{2}\right\}$, where

$$
\begin{aligned}
& r_{1}=\min _{1 \leq k \leq n}\left\{\frac{k^{2} C(n, k)^{2}}{n^{2} C(2 n-2, n-1)}\left|\frac{a_{0}}{a_{k}}\right|\right\}^{1 / k}, \\
& r_{2}=\max _{1 \leq k \leq n}\left\{\frac{n^{2} C(2 n-2, n-1)}{k^{2} C(n, k)^{2}}\left|\frac{a_{n-k}}{a_{n}}\right|\right\}^{1 / k} .
\end{aligned}
$$

3.1. Lemmas. For the proofs of the above theorems, we will need the following lemmas.

Lemma 15. If

$$
C_{n}=\frac{C(2 n, n)}{n+1}
$$

is the nth Catalan number in which $C(2 k, k)$ and $C(n, k)$ are binomial coefficients, then for $n \geq 1$,

$$
\frac{1}{n} \sum_{k=1}^{n} \frac{k C(n, k)^{2}}{n-k+1}=C_{n} .
$$


Proof of Lemma 15. Note that

$$
\begin{aligned}
\frac{1}{n} \sum_{k=1}^{n} \frac{k C(n, k)^{2}}{n-k+1} & =\frac{1}{n} \sum_{k=1}^{n} C(n, k) \frac{k}{n-k+1} C(n, k) \\
& =\frac{1}{n} \sum_{k=1}^{n} C(n, k) \frac{k}{n-k+1} \frac{n !}{k !(n-k) !} \\
& =\frac{1}{n} \sum_{k=1}^{n} C(n, k) \frac{n !}{(k-1) !(n-k+1) !} \\
& =\frac{1}{n} \sum_{k=1}^{n} C(n, k) C(n, k-1)=C_{n} .
\end{aligned}
$$

Lemma 16. If

$$
C_{n}=\frac{C(2 n, n)}{n+1}
$$

is the nth Catalan number in which $C(2 k, k)$ and $C(n, k)$ are binomial coefficients, then for $n \geq 1$,

$$
C_{n+1}=\sum_{k=0}^{n} C(n, 2 k) C_{k} 2^{n-2 k}
$$

Proof of Lemma 16. Note that, by [18, p. 292], we have

$$
C_{n+1}=\sum_{j=0}^{n} C(n, j) M_{j},
$$

where $M_{j}$ is the $j$ th Motzkin number defined by

$$
\begin{aligned}
M_{0} & =M_{1}=M_{-1}=1 ; \\
M_{j+1} & =\frac{2 j+3}{j+3} M_{j}+\frac{3 j}{j+3} M_{j-1}, \quad j \geq 1 .
\end{aligned}
$$

Also, by [18, p. 292], we have

$$
M_{j}=\sum_{k=0}^{j} C(j, 2 k) C_{k} .
$$

Therefore,

$$
\begin{aligned}
C_{n+1} & =\sum_{j=0}^{n} C(n, j) \sum_{k=0}^{j} C(j, 2 k) C_{k} \\
& =\sum_{k=0}^{n} C(n, 2 k) C_{k} 2^{n-2 k} .
\end{aligned}
$$

Lemma 17. If $F_{n}$ is the $n$th Fibonacci number, then for $n \geq 1$,

$$
\sum_{k=1}^{n} F_{2 k}=F_{2 n+1}-1
$$

Proof of Lemma 17. Since $F_{0}=0, F_{1}=1$, and $F_{j}=F_{j-1}+F_{j-2}$ for $j \geq 2$, hence

$$
\begin{gathered}
\sum_{k=1}^{n} F_{2 k}=F_{2}+F_{4}+F_{6}+\cdots+F_{2 n-2}+F_{2 n} \\
=F_{1}+F_{2}+F_{4}+F_{6}+\cdots+F_{2 n-2}+F_{2 n}-F_{1} \\
=F_{3}+F_{4}+F_{6}+\cdots+F_{2 n-2}+F_{2 n}-F_{1} \\
\vdots \\
=F_{2 n-1}+F_{2 n}-F_{1} \\
=F_{2 n+1}-F_{1} .
\end{gathered}
$$

Lemma 18. If $F_{n}$ is the $n$th Fibonacci number, then for $n \geq 1$,

$$
\sum_{k=1}^{n} F_{k}^{2}=F_{n} F_{n+1} .
$$

Proof of Lemma 18. One has

$$
\begin{gathered}
F_{n} F_{n+1}=F_{n}\left(F_{n}+F_{n-1}\right) \\
=F_{n}^{2}+F_{n} F_{n-1} \\
=F_{n}^{2}+F_{n-1}\left(F_{n-1}+F_{n-2}\right) \\
=F_{n}^{2}+F_{n-1}^{2}+F_{n-1} F_{n-2} \\
\vdots \\
=F_{n}^{2}+F_{n-1}^{2}+\cdots+F_{1}^{2} \\
=\sum_{k=1}^{n} F_{k}^{2} .
\end{gathered}
$$

Lemma 19. If

$$
C_{n}=\frac{C(2 n, n)}{n+1}
$$

is the nth Catalan number in which $C(2 k, k)$ and $C(n, k)$ are binomial coefficients, then for $n \geq 1$,

$$
\sum_{k=0}^{n} k^{2} C(n, k)=n(1+n) 2^{n-2} .
$$

Proof of Lemma 19. Note that

$$
\begin{aligned}
\sum_{k=0}^{n} k^{2} C(n, k)= & \sum_{k=1}^{n} k^{2} C(n, k)=\sum_{k=1}^{n} k n C(n-1, k-1) \\
& \text { since } k C(n, k)=n C(n-1, k-1) \\
= & n \sum_{k=0}^{n-1}(k+1) C(n-1, k)
\end{aligned}
$$




$$
\begin{aligned}
& =n \sum_{k=0}^{n-1} k C(n-1, k)+n \sum_{k=0}^{n-1} C(n-1, k) \\
& =n \sum_{k=1}^{n-1} k C(n-1, k)+n \sum_{k=0}^{n-1} C(n-1, k) \\
& =n(n-1) 2^{n-2}+n 2^{n-1}, \\
& \quad \text { ince } \sum_{k=1}^{n-1} k C(n-1, k)=(n-1) 2^{n-2} \\
& =n(1+n) 2^{n-2} .
\end{aligned}
$$

Lemma 20. If

$$
C_{n}=\frac{C(2 n, n)}{n+1}
$$

is the nth Catalan number in which $C(2 k, k)$ and $C(n, k)$ are binomial coefficients, then for $n \geq 1$,

$$
\sum_{k=0}^{n} k C(n, k)^{2}=\frac{n}{2} C(2 n, n) .
$$

Proof of Lemma 20. One has

$$
\begin{aligned}
\sum_{k=0}^{n} k C(n, k)^{2} & =\sum_{k=0}^{n} n C(n-1, k-1) C(n, k) \\
& =\sum_{k=0}^{n} n C(n-1, k-1) C(n, n-k) \\
& =n C(2 n-1, n-1) \\
& =n \frac{(2 n-1) !}{(n-1) ! n !} \\
& =\frac{n}{2} C(2 n, n) .
\end{aligned}
$$

Lemma 21. If

$$
C_{n}=\frac{C(2 n, n)}{n+1}
$$

is the nth Catalan number in which $C(2 k, k)$ and $C(n, k)$ are binomial coefficients, then for $n \geq 1$,

$$
\sum_{k=0}^{n} k^{2} C(n, k)^{2}=n^{2} C(2 n-2, n-1) .
$$

Proof of Lemma 21. One has

$$
\begin{aligned}
\sum_{k=0}^{n} k^{2} C(n, k)^{2} & =\sum_{k=0}^{n} n^{2} C(n-1, k-1)^{2} \\
& =n^{2} \sum_{k=0}^{n} C(n-1, k-1) C(n-1, n-k) \\
& =n^{2} C(2 n-2, n-1) .
\end{aligned}
$$

\subsection{Proofs of the Theorems}

Proof of Theorem 8. By Lemma 15, we have that

$$
\frac{1}{n} \sum_{k=1}^{n} \frac{k C(n, k)^{2}}{n-k+1}=C_{n} \text {. }
$$

If we take

$$
A_{k}=\frac{1}{n} \frac{k C(n, k)^{2}}{(n-k+1) C_{n}},
$$

then $A_{k}>0$ and $\sum_{k=1}^{n} A_{k}=1$. Hence applying Theorem 6 for this set of values of $A_{k}$, we get our desired result.

Proof of Theorem 9. From Lemma 16, we have that

$$
C_{n+1}=\sum_{k=0}^{n} C(n, 2 k) C_{k} 2^{n-2 k}
$$

So $C_{n+1}-2^{n}=\sum_{k=1}^{n} C(n, 2 k) C_{k} 2^{n-2 k}$. If we take

$$
A_{k}=\frac{C(n, 2 k) C_{k} 2^{n-2 k}}{C_{n+1}-2^{n}},
$$

then $A_{k}>0$ and $\sum_{k=1}^{n} A_{k}=1$. Hence by applying Theorem 6 for this set of values of $A_{k}$, Theorem 9 can be proved.

Proof of Theorem 10. From Lemma 17, we have that

$$
\sum_{k=1}^{n} F_{2 k}=F_{2 n+1}-1
$$

If we take

$$
A_{k}=\frac{F_{2 k}}{F_{2 n+1}-1},
$$

then $A_{k}>0$ and $\sum_{k=1}^{n} A_{k}=1$. Hence by applying Theorem 6 for this set of values of $A_{k}$, Theorem 10 can be proved.

Proof of Theorem 11. From Lemma 18, we have that

$$
\sum_{k=1}^{n} F_{k}^{2}=F_{n} F_{n+1}
$$

If we take

$$
A_{k}=\frac{F_{k}^{2}}{F_{n} F_{n+1}},
$$

then $A_{k}>0$ and $\sum_{k=1}^{n} A_{k}=1$. Hence by applying Theorem 6 for this set of values of $A_{k}$, Theorem 11 can be proved.

Proof of Theorem 12. From Lemma 19, we have that

$$
\sum_{k=0}^{n} k^{2} C(n, k)=n(1+n) 2^{n-2} .
$$

If we take

$$
A_{k}=\frac{k^{2} C(n, k)}{n(1+n) 2^{n-2}},
$$

then $A_{k}>0$ and $\sum_{k=1}^{n} A_{k}=1$. Hence by applying Theorem 6 for this set of values of $A_{k}$, Theorem 12 can be proved. 
TABLE 1

\begin{tabular}{lccc}
\hline Theorems & $r_{1}$ & $r_{2}$ & Area of the annulus \\
\hline 5 & 0.4641 & 1.6984 & 8.382 \\
7 & 0.6542 & 1.205 & 3.2159 \\
8 & 0.5192 & 1.5183 & 6.395 \\
10 & 0.5833 & 1.20 & 3.4549 \\
11 & 0.7757 & 1.0164 & 1.3553 \\
12 & 0.6403 & 1.2313 & 3.4748 \\
14 & 0.4886 & 1.6134 & 7.428 \\
\hline
\end{tabular}

Proof of Theorem 13. From Lemma 20, we have that

$$
\sum_{k=0}^{n} k C(n, k)^{2}=\frac{n}{2} C(2 n, n) .
$$

If we take

$$
A_{k}=\frac{k C(n, k)^{2}}{(n / 2) C(2 n, n)},
$$

then $A_{k}>0$ and $\sum_{k=1}^{n} A_{k}=1$. Hence by applying Theorem 6 for this set of values of $A_{k}$, Theorem 13 can be proved.

Proof of Theorem 14. From Lemma 21, we have that

$$
\sum_{k=0}^{n} k^{2} C(n, k)^{2}=n^{2} C(2 n-2, n-1) .
$$

If we take

$$
A_{k}=\frac{k^{2} C(n, k)^{2}}{n^{2} C(2 n-2, n-1)},
$$

then $A_{k}>0$ and $\sum_{k=1}^{n} A_{k}=1$. Hence by applying Theorem 6 for this set of values of $A_{k}$, Theorem 14 can be proved.

\section{Computational Results and Analysis}

In this section, we present two examples of polynomials in order to compare our theorems with some of the above stated known theorems and show that for these polynomials our theorems give better bounds than those obtainable by these known theorems.

Example 1. Consider the polynomial $p(z)=z^{3}+0.1 z^{2}+0.1 z+$ 0.7 .

Table 1 suggests that our Theorem 11 gives significantly better bounds than those obtained from any result, including Theorems 5 and 7. As can be seen, the area of the annulus obtained by Theorem 11 is about $16.17 \%$ of the area of the annulus obtained by Theorem 5 and about $42.14 \%$ of the area obtained by Theorem 7 . The inner radius obtained from Theorem 11 is almost $167 \%$ of the inner radius obtained from Theorem 5, and similarly the outer radius obtained from Theorem 11 is almost $59.84 \%$ of the outer radius obtained from Theorem 5 .
TABLE 2

\begin{tabular}{lccc}
\hline Theorems & $r_{1}$ & $r_{2}$ & Area of the annulus \\
\hline 5 & $5.56 \times 10^{-4}$ & 1.158 & 4.2125 \\
7 & 0.0011 & 1.5608 & 7.6529 \\
8 & $8.21 \times 10^{-5}$ & 1.1036 & 3.8265 \\
12 & $7.18 \times 10^{-5}$ & 1.3191 & 5.4664 \\
13 & $1.37 \times 10^{-4}$ & 1.1626 & 4.2464 \\
14 & $4.93 \times 10^{-5}$ & 1.1264 & 3.9859 \\
\hline
\end{tabular}

Example 2. Consider the polynomial

$$
\begin{aligned}
p(z)= & z^{5}+0.006 z^{4}+0.29 z^{3}+0.29 z^{2}+0.29 z \\
& +0.001
\end{aligned}
$$

As shown in Table 2, the best upper bound of the annular region containing all the zeros of the polynomial $p(z)$ comes from Theorem 8, which also gives the smallest area of the annulus containing all the zeros. The area of the annulus obtained by Theorem 8 is about $90.84 \%$ of the area of the annulus obtained by Theorem 5 and about $50 \%$ of the area of the annulus obtained by Theorem 7 .

\section{Data Availability}

No data from any source has been used in order to prepare this paper, except some mathematical results which have been duly cited in the paper.

\section{Conflicts of Interest}

The authors declare that there are no conflicts of interest regarding the publication of this paper.

\section{References}

[1] H. Anai and K. Horimoto, Algebraic Biology 2005, Proceedings of the First Conference on Algebraic Biology, Tokyo, Japan, 2005.

[2] C. Bissel, Control Engineering, CRC Press, 2nd edition, 2009.

[3] N. K. Govil and E. R. Nwaeze, "On geometry of the zeros of a polynomial," in Computation, Cryptography, and Network Security, N. J. Daras and M. Th. Rassias, Eds., pp. 253-287, Springer, Cham, Switzerland, 2015.

[4] L. Pachter and B. Sturmdfels, Algebraic Statistics for Computational Biology, Cambridge University Press, 2005.

[5] O. Aberth, "Iteration methods for finding all zeros of a polynomial simultaneously," Mathematics of Computation, vol. 27, pp. 339-344, 1973.

[6] L. W. Ehrlich, "A modified Newton method for polynomials," Communications of the ACM, vol. 10, no. 2, pp. 107-108, 1967.

[7] G. V. Milovanović and M. S. Petković, "On computational efficiency of the iterative methods for the simultaneous approximation of polynomial zeros," ACM Transactions on Mathematical Software, vol. 12, no. 4, pp. 295-306, 1986.

[8] M. S. Petković, "A highly efficient zero-solver of very fast convergence," Applied Mathematics and Computation, vol. 205, no. 1, pp. 298-302, 2008. 
[9] K. F. Gauss, Beiträge zur Theorie der algebraischen Gleichungen, vol. 4, Abhandlungen der Königlichen Gesellschaft der Wissenschaften zu Göttingen, 1850, Ges. Werke, vol. 3, pp. 73-102, 1850.

[10] A. L. Cauchy, Excercises de Mathématique, IV Année de Bure Freres, Paris, France, 1829.

[11] J. L. Diaz-Barrero, "An annulus for the zeros of polynomials," Journal of Mathematical Analysis and Applications, vol. 273, no. 2, pp. 349-352, 2002.

[12] S.-H. Kim, "On the moduli of the zeros of a polynomial," The American Mathematical Monthly, vol. 112, no. 10, pp. 924-925, 2005.

[13] M. Bidkham and E. Shashahani, "An annulus for the zeros of polynomials," Applied Mathematics Letters, vol. 24, no. 2, pp. 122-125, 2011.

[14] N. A. Rather and S. G. Mattoo, "On annulus containing all the zeros of a polynomial," Applied Mathematics E-Notes, vol. 13, pp. 155-159, 2013.

[15] A. Dalal and N. K. Govil, "On region containing all the zeros of a polynomial," Applied Mathematics and Computation, vol. 219, no. 17, pp. 9609-9614, 2013.

[16] A. Dalal and N. K. Govil, "Annulus containing all the zeros of a polynomial," Applied Mathematics and Computation, vol. 249, pp. 429-435, 2014.

[17] A. Dalal and N. K. Govil, "On comparison of annuli containing all the zeros of a polynomial," Applicable Analysis and Discrete Mathematics, vol. 11, no. 1, pp. 232-241, 2017.

[18] R. Donaghey and L. W. Shapiro, "Motzkin numbers," Journal of Combinatorial Theory, Series A, vol. 23, no. 3, pp. 291-301, 1977. 


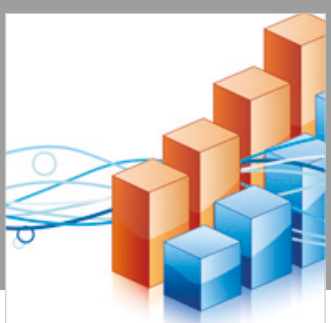

Advances in

Operations Research

\section{-n-m}
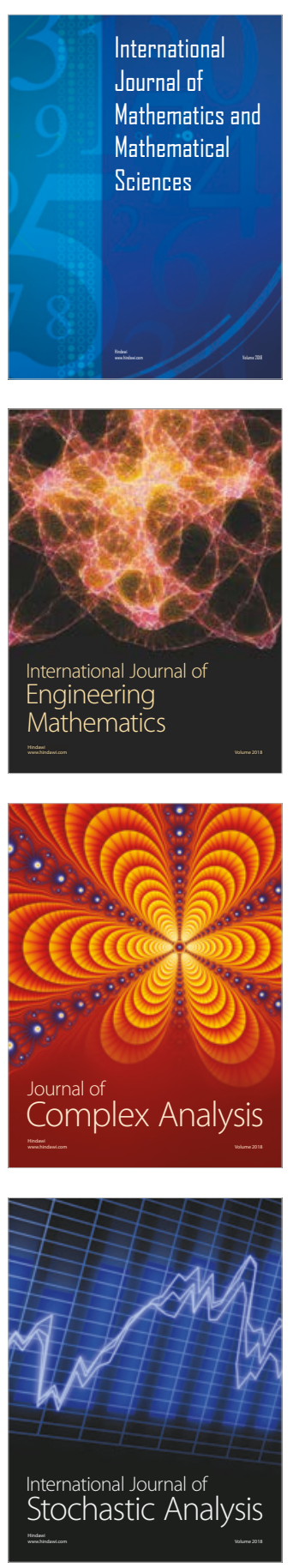
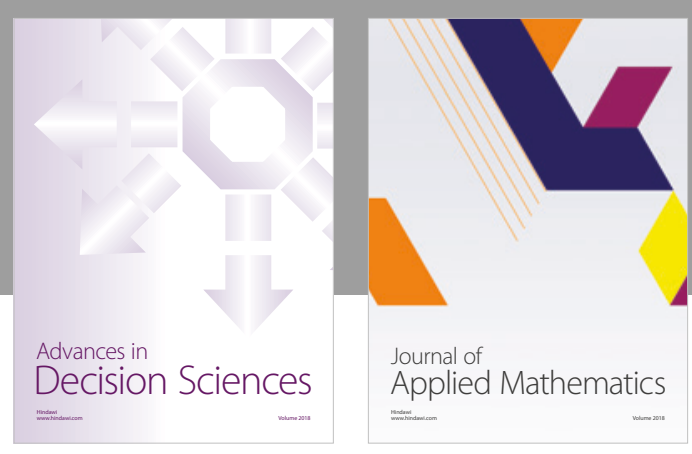

Journal of

Applied Mathematics
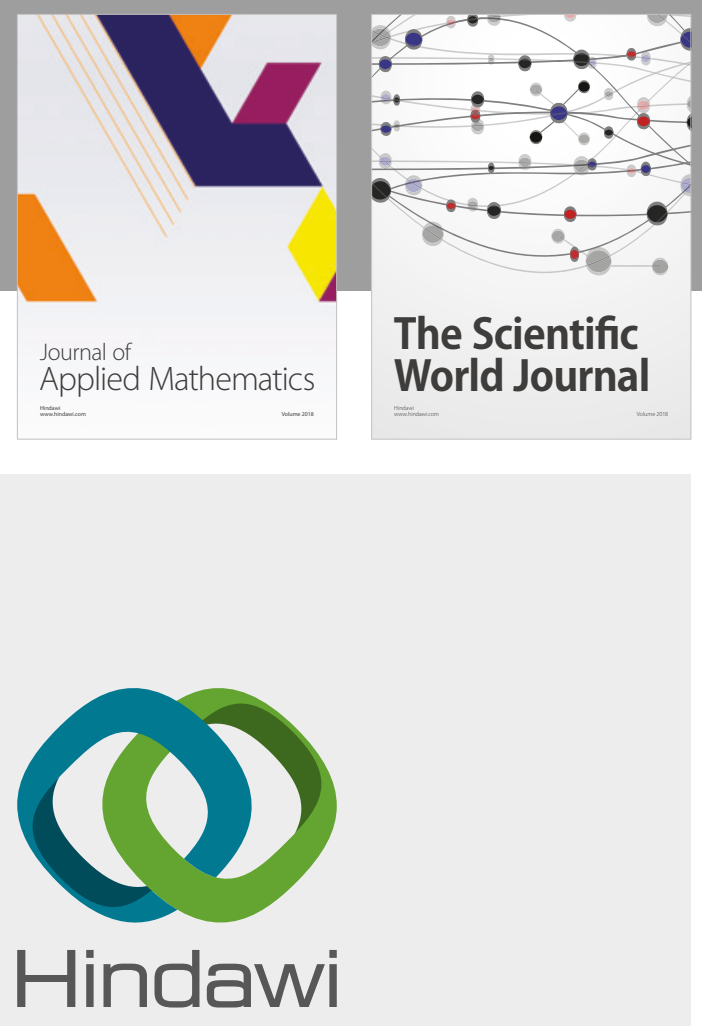

Submit your manuscripts at

www.hindawi.com

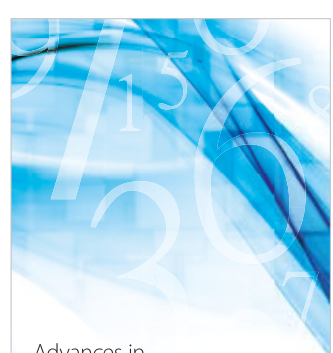

Advances in
Numerical Analysis
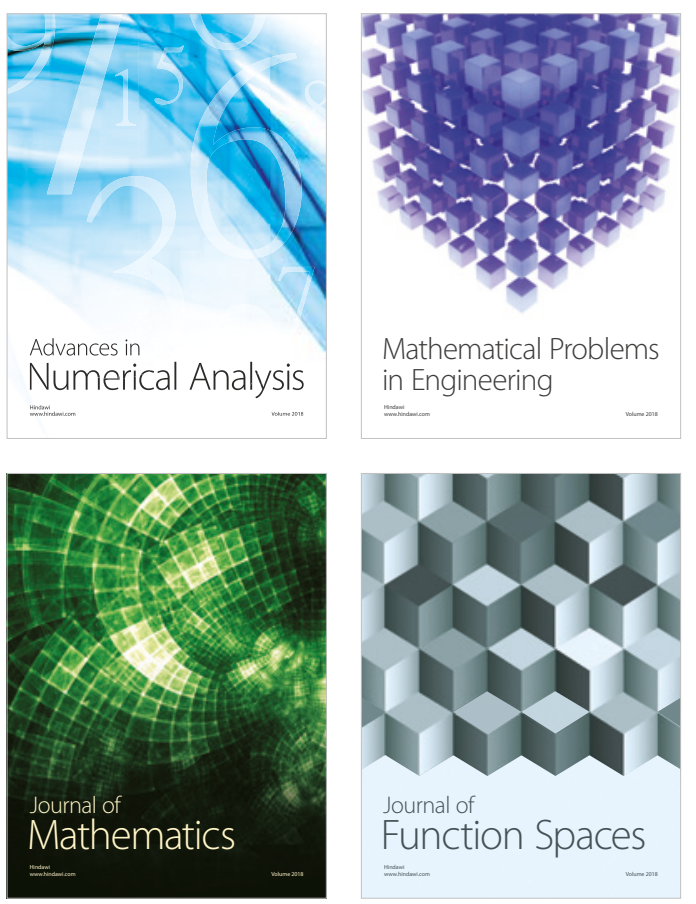

Mathematical Problems in Engineering

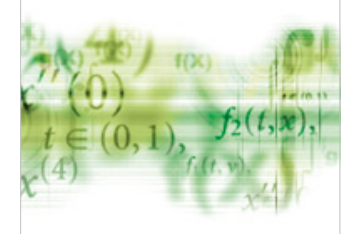

International Journal of

Differential Equations

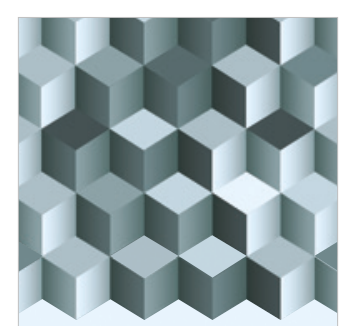

Journal of

Function Spaces

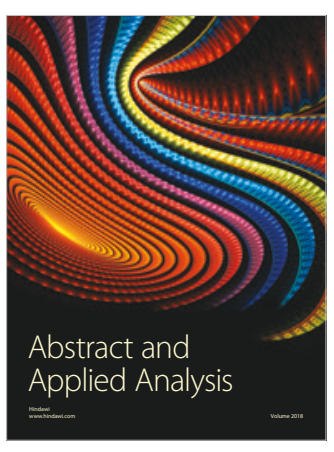

The Scientific

World Journal

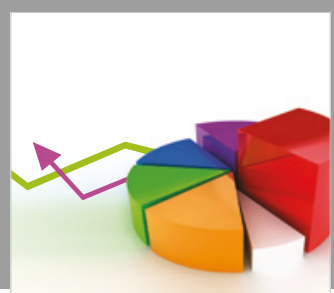

Journal of

Probability and Statistics
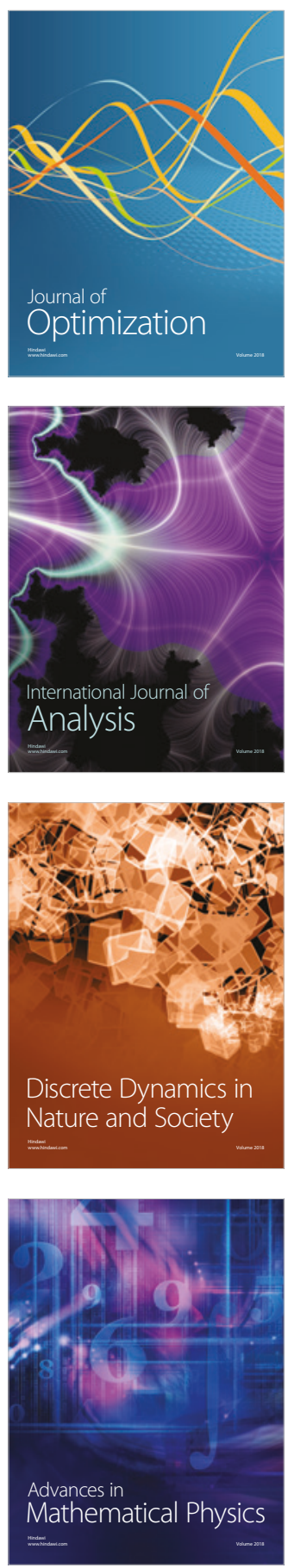